\title{
Fuzzy Chromatic Polynomial of Fuzzy Graphs with Crisp and Fuzzy Vertices Using $\alpha$-Cuts
}

\author{
Mamo Abebe Ashebo $(i)$ and V. N. Srinivasa Rao Repalle \\ Department of Mathematics, Wollega University, Nekemte, Ethiopia \\ Correspondence should be addressed to Mamo Abebe Ashebo; mamoabebe37@gmail.com
}

Received 24 January 2019; Revised 28 March 2019; Accepted 2 April 2019; Published 2 May 2019

Academic Editor: Antonin Dvorák

Copyright (c) 2019 Mamo Abebe Ashebo and V. N. Srinivasa Rao Repalle. This is an open access article distributed under the Creative Commons Attribution License, which permits unrestricted use, distribution, and reproduction in any medium, provided the original work is properly cited.

\begin{abstract}
Coloring of fuzzy graphs has many real life applications in combinatorial optimization problems like traffic light system, exam scheduling, register allocation, etc. In this paper, the concept of fuzzy chromatic polynomial of fuzzy graph is introduced and defined based on $\alpha$-cuts of fuzzy graph. Two different types of fuzziness to fuzzy graph are considered in the paper. The first type was fuzzy graph with crisp vertex set and fuzzy edge set and the second type was fuzzy graph with fuzzy vertex set and fuzzy edge set. Depending on this, the fuzzy chromatic polynomials for some fuzzy graphs are discussed. Some interesting remarks on fuzzy chromatic polynomial of fuzzy graphs have been derived. Further, some results related to the concept are proved. Lastly, fuzzy chromatic polynomials for complete fuzzy graphs and fuzzy cycles are studied and some results are obtained.
\end{abstract}

\section{Introduction}

Nowadays, many real world problems cannot be properly modeled by a crisp graph theory, since the problems contain uncertain information. The fuzzy set theory, anticipated by Zadeh [1], is used to handle the phenomena of uncertainty in real life situation. A lot of works have been done in fuzzy shortest path problems using type 1 fuzzy set in [2-5]. Dey et al. [6] introduced interval type 2 fuzzy set in the fuzzy shortest path problems. Recently, in [7], the authors proposed a genetic algorithm for solving fuzzy shortest path problem with interval type 2 fuzzy arc lengths. Some researchers also used the fuzzy set theory to touch the uncertainty in crisp graphs. Kaufmann [8] proposed the first definition of fuzzy graph in 1973, based on Zadeh's fuzzy relations. Later, Rosenfeld [9] introduced another elaborated definition of fuzzy graph with fuzzy vertex set and fuzzy edge set in 1975. He developed the theory of fuzzy graph. After that, Bhattacharya [10] has established some connectivity concepts regarding fuzzy cut nodes and fuzzy bridges. Bhutani [11] has studied automorphisms on fuzzy graphs and certain properties of complete fuzzy graphs. Also, Mordeson and Nair [12] introduced cycles and cocycles of fuzzy graphs. Several authors including Sunitha and Vijayakumar [13, 14],
Bhutani and Rosenfeld [15], Mathew and Sunitha [16], Akram [17], and Akram and Dudek [18] have introduced numerous concepts in fuzzy graphs. Fuzzy graph theory has several applications in various fields like clustering analysis, database theory, network analysis, information theory, etc. [19].

Coloring of fuzzy graphs plays a vital role in theory and practical applications. It is mainly studied in combinatorial optimization problems like traffic light control, exam scheduling, register allocation, etc. [20]. Fuzzy coloring of a fuzzy graph was defined by authors Eslahchi and Onagh in 2004 and later developed by them as fuzzy vertex coloring [21] in 2006. Another approach of coloring of fuzzy graphs was introduced by Munoz et al. [22]. The authors have defined the chromatic number of a fuzzy graph. Incorporating the above two approaches of coloring of fuzzy graph, Kishore and Sunitha [23] introduced chromatic number of fuzzy graphs and developed algorithm. Dey and Pal [24] introduced the vertex coloring of a fuzzy graph using $\alpha$-cuts. In [25], they have used the vertex coloring of fuzzy graph to classify the accidental zone of a traffic control. Further, in [26], the authors proposed genetic algorithm to find the robust solutions for fuzzy robust coloring problem. The authors, Ananthanarayanan and Lavanya [20], introduced fuzzy chromatic number and fuzzy total chromatic number 
of a fuzzy graph using $\alpha$-cuts. Rosyida et al. [27] proposed a new approach to determine fuzzy chromatic number of fuzzy graph through its $\delta$-chromatic number. Samanta et al. [28] also introduced a new concept for coloring of fuzzy graphs using fuzzy color. Lately, Dey et al. [29] introduced the concept of vertex and edge coloring of vague graphs which are the generalized structure of fuzzy graphs.

In the literature, to the best of our knowledge, there is no study on the fuzzy chromatic polynomial of fuzzy graphs. Therefore, in this paper, we consider the chromatic polynomial in fuzzy graph, called fuzzy chromatic polynomial of fuzzy graph. Based on this, we define the concept of fuzzy chromatic polynomial of a fuzzy graph using $\alpha$ cuts of fuzzy graph. Also, we determine the fuzzy chromatic polynomial for fuzzy graphs with crisp and fuzzy vertex set. To determine the fuzzy chromatic polynomial, the classical method for computing the chromatic polynomial of crisp graph is used. Next, we derive some interesting remarks on fuzzy chromatic polynomial of fuzzy graph with crisp and fuzzy vertices. Further, we prove more elegant results on fuzzy chromatic polynomial of fuzzy graphs. Finally, we study a fuzzy chromatic polynomial for complete fuzzy graphs and fuzzy cycles.

The rest of paper is organized as follows. In Section 2, some basic definitions and elementary concepts of fuzzy set, fuzzy graph, and coloring of fuzzy graphs are reviewed. In Section 3, fuzzy chromatic polynomial of a fuzzy graph using $\alpha$-cut of fuzzy graph is defined. Also, fuzzy chromatic polynomials for fuzzy graphs with crisp and fuzzy vertices are determined. In Section 4, more results on fuzzy chromatic polynomials are proved. Furthermore, fuzzy chromatic polynomial for complete fuzzy graphs and fuzzy cycles are studied. Finally, the paper is concluded in Section 5.

\section{Preliminaries}

In this section, some basic aspects that are necessary for this paper are included. These preliminaries are given in three subsections.

2.1. Basic Definitions and Concepts on Vertex Coloring and Chromatic Polynomial. In this subsection some basic definitions and concepts of vertex coloring and chromatic polynomials are reviewed [30, 31].

Definition 1. Let $\mathrm{G}=(\mathrm{V}, \mathrm{E})$ be a graph. A vertex-coloring of $G$ is an assignment of a color to each of the vertices of $\mathrm{G}$ in such a way that adjacent vertices are assigned different colors. If the colors are chosen from a set of $k$ colors, then the vertex-coloring is called a $k$-vertex-coloring, abbreviated to $k$-coloring, whether or not all $k$ colors are used.

Definition 2. If $\mathrm{G}$ has a $k$-coloring, then $G$ is said to be $k$ colorable.

Definition 3. The smallest $k$, such that $\mathrm{G}$ is $k$-colorable, is called the chromatic number of $\mathrm{G}$, denoted by $\chi(\mathrm{G})$.
Definition 4. Let $\mathrm{G}$ be a simple graph. The chromatic polynomial of $G$ is the number of ways we can achieve a proper coloring on the vertices of $\mathrm{G}$ with the given $k$ colors and it is denoted by $P(\mathrm{G}, k)$. It is a monic polynomial in $k$ with integer coefficients, whose degree is the number of vertices of $\mathrm{G}$.

If we are given an arbitrary simple graph, it is usually difficult to obtain its chromatic polynomial by examining the structure of a graph (by inspection). The following theorem gives us a systematic method for obtaining the chromatic polynomial of a simple graph in terms of the chromatic polynomial of null graphs.

Theorem 5. Let $\mathrm{G}$ be a simple graph, and let $\mathrm{G}-\mathrm{e}$ and $\mathrm{G} / \mathrm{e}$ be the graphs obtained from $\mathrm{G}$ by deleting and contracting an edge e. Then

$$
P(G, k)=P(G-e, k)-P(G / e, k)
$$

2.2. Basic Definitions on Fuzzy Set and Fuzzy Graphs. In this subsection, some basic definitions on fuzzy set and fuzzy graphs are reviewed $[1,9,19,32]$.

Definition 6. A fuzzy set $A$ defined on a nonempty set $X$ is the family $A=\left\{\left(x, \mu_{A}(x)\right) \mid x \in X\right\}$, where $\mu_{A}: X \longrightarrow I$ is the membership function. In classical fuzzy set theory the set $I$ is usually defined as the interval $[0,1]$ such that

$$
\mu_{A}(x)= \begin{cases}1, & x \in A \\ 0, & x \notin A\end{cases}
$$

It takes any intermediate value between 0 and 1 represents the degree in which $x \in A$. The set $I$ could be discrete set of the form $I=\{0,1, \ldots, k\}$ where $\mu_{A}(x)<\mu_{A}\left(x^{\prime}\right)$ indicates that the degree of membership of $x$ to $A$ is lower than the degree of membership of $x^{\prime}$.

Definition 7. $\alpha$-cut set of fuzzy set $A$ is defined as $A_{\alpha}$ is made up of members whose membership is not less than $\alpha, A_{\alpha}=$ $\left\{x \in X \mid \mu_{A}(x) \geq \alpha\right\}$. $\alpha$-cut set of fuzzy set is crisp set.

Definition 8. A fuzzy graph $G=(V, \sigma, \mu)$ is an algebraic structure of nonempty set $V$ together with a pair of functions $\sigma: V \longrightarrow[0,1]$ and $\mu: V \times V \longrightarrow[0,1]$ such that for all $u, v \in$ $V, \mu(u, v) \leq \sigma(u) \wedge \sigma(v)$ and $\mu$ is a symmetric fuzzy relation on $\sigma$. Here $\sigma(u)$ and $\mu(u, v)$ represent the membership values of the vertex $u$ and of the edge $(u, v)$ in $G$, respectively.

In this paper, we denote $u \wedge v=\min \{u, v\}$ and $u \vee$ $v=\max \{u, v\}$. Here, we considered fuzzy graph $G$ is simple (with no loops and parallel edges), finite, and undirected. $\mu$ is reflective (that is, $\mu(v, v)=\sigma(v)$, for all $v \in V$ ) and symmetric (that is, $\mu(u, v)=\mu(v, u)$, for all $(u, v) \in E$ ).

Note that a fuzzy graph is a generalization of crisp graph in which $\sigma(v)=1$ for all $v \in V$ and $\mu(u, v)=1$ if $(u, v) \in E$ and $\mu(u, v)=0$ if $(u, v) \notin E$. So, all the crisp graphs are fuzzy graphs but all fuzzy graphs are not crisp graphs. 
Definition 9. The fuzzy graph $G^{\prime}=\left(V, \sigma^{\prime}, \mu^{\prime}\right)$ is called a fuzzy subgraph of $G$ if for each two elements $u, v \in V$, we have $\sigma^{\prime}(u) \leq \sigma(u)$ and $\mu^{\prime}(u, v) \leq \mu(u, v)$.

Definition 10. The fuzzy graph $G=(V, \sigma, \mu)$ is called connected if, for every two elements $\mathrm{u}, v \in V$, there exists a sequence of elements $u_{0}, u_{1}, \ldots, u_{m}$ such that $u_{0}=u, u_{m}=v$, and $\mu\left(u_{i}, u_{i+1}\right)>0,(0 \leq i \leq m-1)$.

Definition 11. For any fuzzy graph $G=(V, \sigma, \mu)$, let $L=$ $\{\sigma(u)>0 \mid u \in V\} \cup\{\mu(u, v)>0 \mid u \neq v, u, v \in V\}$ with $k$ elements. Now assume $L=\left\{\alpha_{1}, \alpha_{2}, \ldots, \alpha_{k}\right\}$ such that $\alpha_{1}<\alpha_{2}<\cdots<\alpha_{k}$. The sequence $\left\{\alpha_{1}, \alpha_{2}, \ldots, \alpha_{k}\right\}$ and the set $L$ are called the fundamental sequence and the fundamental set (or level set) of $G$, respectively.

Definition 12. The underlying crisp graph $G^{*}=\left(V^{*}, E^{*}\right)$ of a fuzzy graph $G=(V, \sigma, \mu)$ is such that $V^{*}=\{u \in V \mid \sigma(u)>0\}$ and $E^{*}=\{(u, v) \mid \mu(u, v)>0\}$.

Definition 13. For $\alpha \in[0,1], \alpha$-cut graph of a fuzzy graph $G=$ $(V, \sigma, \mu)$ is a crisp graph $G_{\alpha}=\left(V_{\alpha}, E_{\alpha}\right)$ such that $V_{\alpha}=\{u \in V \mid$ $\sigma(u) \geq \alpha\}$ and $E_{\alpha}=\{(u, v) \mid \mu(u, v) \geq \alpha\}$. It is obvious that a fuzzy graph will have a finite number of different $\alpha$-cuts.

2.3. Basic Definitions in Fuzzy Coloring of Fuzzy Graphs. The concept of chromatic number of fuzzy graph was introduced by Munoz et al. [22]. The authors considered fuzzy graphs with crisp vertex set, that is, fuzzy graphs, for which $\sigma(v)=1$ for all $v \in V$ and edges with membership degree in $[0,1]$.

Definition 14 (see [22]). If $G=(V, \mu)$ is such a fuzzy graph where $V=\{1,2,3 \ldots n\}$ and $\mu$ is a fuzzy number on the set of all subsets of $V \times V$, assume $I=A \cup\{0\}$, where $A=\left\{\alpha_{1}<\right.$ $\left.\alpha_{2}<\ldots<\alpha_{k}\right\}$ is the fundamental set (level set) of $G$. For each $\alpha \in I, G_{\alpha}$ denote the crisp graph $\mathrm{G}_{\alpha}=\left(V, E_{\alpha}\right)$, where $E_{\alpha}=\{i j \mid 1 \leq i<j \leq n, \mu(i, j) \geq \alpha\}$ and $\chi_{\alpha}=\chi\left(G_{\alpha}\right)$ denote the chromatic number of crisp graph $G_{\alpha}$.

By this definition the chromatic number of fuzzy graphs $\mathrm{G}$ is the fuzzy number $\chi(G)=\{(i, v(i)) / i \in X\}$, where $v(i)=$ $\max \left\{\alpha \in I \mid i \in A_{\alpha}\right\}$ and $A_{\alpha}=\left\{1,2,3 \ldots, \chi_{\alpha}\right\}$.

Later Eslahchi and Onagh [21] introduced fuzzy vertex coloring of fuzzy graph. They defined fuzzy chromatic number as the least value of $k$ for which the fuzzy graph $G$ has $k$-fuzzy coloring as follows.

Definition 15 (see [21]). A family $\Gamma=\left\{\gamma_{1}, \gamma_{2}, \ldots, \gamma_{k}\right\}$ of fuzzy sets on a set $V$ is called a $k$-fuzzy coloring of $G=(V, \sigma, \mu)$ if

(i) $\vee \Gamma=\sigma$,

(ii) $\gamma_{i} \wedge \gamma_{j}=0$,

(iii) for every strong edge $(x, y)$ (i.e., $\mu(x, y)>0)$ of $G$, $\min \left\{\gamma_{i}(x), \gamma_{i}(y)\right\}=0(1 \leq i \leq k)$.

Definition 16 (see [21]). The minimum number $k$ for which there exists a $k$-fuzzy coloring is called the fuzzy chromatic number of $G$, denoted as $\chi^{f}(G)$.
Incorporating the features of the above two definitions, Kishore and Sunitha [23] modified the chromatic number $\chi(G)$ of fuzzy graph as follows.

Definition 17 (see [23]). For each $\alpha \in I, G_{\alpha}$ denote the crisp graph $G_{\alpha}=\left(\sigma_{\alpha}, \mu_{\alpha}\right)$ and $\chi_{\alpha}=\chi\left(G_{\alpha}\right)$ denote the chromatic number of crisp graph $G_{\alpha}$. The chromatic number of fuzzy graph $G=(V, \sigma, \mu)$ is the number $\chi(G)=\max \left\{\chi\left(G_{\alpha}\right) \mid \alpha \epsilon\right.$ A\}.

\section{Fuzzy Chromatic Polynomial of a Fuzzy Graph}

A fuzzy chromatic polynomial is a polynomial which is associated with the fuzzy coloring of fuzzy graphs. Therefore, chromatic polynomial in fuzzy graph is called fuzzy chromatic polynomial of fuzzy graph. In this section, we define the concept of fuzzy chromatic polynomial of a fuzzy graph based on $\alpha$-cuts of fuzzy graph which are crisp graphs. Furthermore, we determine the fuzzy chromatic polynomials for some fuzzy graphs with crisp and fuzzy vertices.

Crisp vertex coloring and the chromatic polynomial of $\alpha$ cut graph of the fuzzy graph are defined as follows.

Definition 18. Let $G=(V, \sigma, \mu)$ be a fuzzy graph and $G_{\alpha}$ denote $\alpha$-cut graph of the fuzzy graph $G$ which is a crisp graph $G_{\alpha}=\left(V_{\alpha}, E_{\alpha}\right), \alpha \in[0,1]$. A function $C_{\alpha}^{k}: V_{\alpha} \longrightarrow\{1,2, \ldots, k\}$, $k \in \mathbb{N}$ is called a $k$-coloring (crisp vertex coloring) of $G_{\alpha}$ if $C_{\alpha}^{k}\left(v_{i}\right) \neq C_{\alpha}^{k}\left(v_{j}\right)$ whenever the vertices $v_{i}$ and $v_{j}$ are adjacent in $G_{\alpha}$.

Definition 19. The number of distinct k-coloring on the vertices of $G_{\alpha}$ is called the chromatic polynomial of $G_{\alpha}$. It is denoted by $P\left(G_{\alpha}, k\right)$.

Let $\left\{G_{\alpha} \mid \alpha \in I\right\}$ be the family of $\alpha$-cuts sets of $G$, where the $\alpha$-cut of a fuzzy graph is the crisp graph $G_{\alpha}$. Hence, any crisp k-coloring $C_{\alpha}^{k}$ can be defined on the vertex set of $G_{\alpha}$. The k-coloring function of the fuzzy graph $G$ is defined through this sequence. For each $\alpha \in I$, let the fuzzy chromatic polynomial of $G$ be defined through a monotone family of sets.

Fuzzy chromatic polynomial of a fuzzy graph is defined as follows.

Definition 20. Let $G$ be a fuzzy graph. The fuzzy chromatic polynomial of $G$ is defined as the chromatic polynomial of its crisp graphs $G_{\alpha}$, for $\alpha \in I$. It is denoted by $P_{\alpha}^{f}(G, k)$.

That is, $P_{\alpha}^{f}(G, k)=P\left(G_{\alpha}, k\right), \forall \alpha \in I$.

3.1. Fuzzy Chromatic Polynomial of Fuzzy Graph with Crisp Vertices. In this subsection, we present fuzzy chromatic polynomial of fuzzy graphs with crisp vertices and fuzzy edges.

A fuzzy graph $G$ with crisp vertices and fuzzy edges, and $\alpha$-cut graph of $G$ are defined as follows. 
Definition 21. A fuzzy graph is defined as a pair $G=(V, \mu)$ such that

(1) $\mathrm{V}$ is the crisp set of vertices (that is, $\sigma(u)=1, \forall u \in V$ );

(2) the function $\mu: V \times V \rightarrow[0,1]$ is defined by $\mu(u, v) \leq \sigma(u) \wedge \sigma(v)$, for all $u, v \in V$.

Definition 22. Let $G=(V, \mu)$ be a fuzzy graph. For $\alpha \in I, \alpha$ cut graph of the fuzzy graph $G$ is defined as the crisp graph $G_{\alpha}=\left(V, E_{\alpha}\right)$, where $E_{\alpha}=\{(u, v), u, v \in V \mid \mu(u, v) \geq \alpha\}$.

Example 23. Consider the fuzzy graph $G$ with crisp vertices and fuzzy edges in Figure 1.

In $G$, we consider $I=\{0,0.2,0.3,0.5,0.8,1\}$; for each $\alpha \in I$, we have a crisp graph $G_{\alpha}$ and its chromatic polynomial which is the fuzzy chromatic polynomial of the fuzzy graph $G$ is obtained (see Figure 2). (The integers in the brackets denote the number of ways of coloring the vertices.)

Remark 24. The fuzzy chromatic polynomial depends on the values of $\alpha$, which means the fuzzy chromatic polynomial varies for the same fuzzy graph $G$ for different values of $\alpha$.

For the fuzzy graph $G$ in Example 23, the fuzzy chromatic polynomial varies for different values of $\alpha$ as shown below:

$$
P_{\alpha}^{f}(G, k)= \begin{cases}k(k-1)(k-2)(k-3), & \alpha=0 \\ k(k-1)(k-2)^{2}, & \alpha=0.2 \\ k(k-1)^{2}(k-2), & \alpha=0.3 \\ k(k-1)^{3}, & \alpha=0.5 \\ k^{2}(k-1)^{2}, & \alpha=0.8 \\ k^{4}, & \alpha=1\end{cases}
$$

Remark 25. The fuzzy chromatic polynomial of a fuzzy graph does not need to be decreased when the value of $\alpha$ increases. Let us consider $\alpha_{1}=0.2, \alpha_{2}=0.3, \alpha_{3}=0.5, \alpha_{4}=0.8$, and $\alpha_{5}=1$ for the fuzzy graph $G$ in Example 23, but $P_{\alpha_{i}}^{f}(G, k)$, $k \geq \chi_{\alpha_{i}}$, where $\chi_{\alpha_{i}}$ is the chromatic number of the crisp graph $G_{\alpha_{i}} \mathrm{i}=1,2,3,4,5$ is not decreased as $\left\{\alpha_{i}\right\}$ increases. For instance, $P_{\alpha_{1}}^{f}\left(G, \chi_{\alpha_{1}}\right)=6, P_{\alpha_{2}}^{f}\left(G, \chi_{\alpha_{2}}\right)=12, P_{\alpha_{3}}^{f}\left(G, \chi_{\alpha_{3}}\right)=2$, $P_{\alpha_{4}}^{f}\left(G, \chi_{\alpha_{4}}\right)=4$, and $P_{\alpha_{5}}^{f}\left(G, \chi_{\alpha_{5}}\right)=1$.

Remark 26. Let $G$ be a fuzzy graph with $n$ vertices. Then for $G$ with crisp vertices and fuzzy edges and for $\alpha=1, P_{\alpha}^{f}(G, k)$ is not always $P\left(N_{n}, k\right)$, where $N_{n}$ is the null crisp graph with $\mathrm{n}$ vertices which is clear from the following example.

Example 27. Consider the fuzzy graph $G$ given in Figure 3(a). In Figure 3(a), for $\alpha=1, G_{\alpha}$ is a crisp graph in Figure 3(b). But $G_{\alpha}$ is not a null crisp graph with three vertices. Thus, $P_{\alpha}^{f}(G, k) \neq P\left(N_{3}, k\right)$.

The following result gives the degree of fuzzy chromatic polynomial of fuzzy graph with crisp vertices and fuzzy edges are equal to the number of vertices in crisp vertex set.



FIGURE 1: The fuzzy graph $G$ with crisp vertices and fuzzy edges.

Lemma 28. Let $G=(V, \mu)$ be a fuzzy graph with crisp vertices and fuzzy edges. Then the degree of $P_{\alpha}^{f}(G, k)=|V|$, for all $\alpha \in$ I.

Proof. Let $G=(V, \mu)$ be a fuzzy graph with crisp vertices and fuzzy edges. Now, $G_{\alpha}=\left(V, E_{\alpha}\right)$, where $E_{\alpha}=\{(u, v)$ | $\mu(u, v) \geq \alpha$. We know that the degree of the chromatic polynomial of a crisp graph $G_{\alpha}$ is the number of vertices of $G_{\alpha}$. That is, the degree of $P\left(G_{\alpha}, k\right)=|V|$. By Definition 20, we have $P_{\alpha}^{f}(G, k)=P\left(G_{\alpha}, k\right)$, for $\alpha \in I$. From this it follows that the degree of $P_{\alpha}^{f}(G, k)$ is equal to the degree of $P\left(G_{\alpha}, k\right)$. Therefore, $P_{\alpha}^{f}(G, k)=|V|$.

3.2. Fuzzy Chromatic Polynomial of Fuzzy Graph with Fuzzy Vertices. In Section 3.1, we introduced the fuzzy chromatic polynomial of fuzzy graph $G=(V, \mu)$, where $V$ is crisp set vertices and $E$ is fuzzy set of edges. In this subsection, we present fuzzy chromatic polynomial of fuzzy graphs with fuzzy vertex set and fuzzy edge set. Here, we use Definition 8 for fuzzy graph and Definition 13 for $\alpha$-cut graph which are presented in Section 2.

Example 29. Consider a fuzzy graph $G$ with fuzzy vertex set and fuzzy edge set in Figure 4

In $G$, we consider $I=\{0,0.2,0.3,0.4,0.6,0.8\}$; for each $\alpha \in I$, we have a crisp graph $G_{\alpha}$ and its chromatic polynomial which is the fuzzy chromatic polynomial of the fuzzy graph $G$ is obtained (see Figure 5). (The integers in the brackets denote the number of ways of coloring the vertices.)

Remark 30. The fuzzy chromatic polynomial varies for the same fuzzy graph $G$ for different values of $\alpha$. It is clear from Example 29. Here, the fuzzy chromatic polynomial of the fuzzy graph in Example 29 is given below.

$$
\begin{aligned}
& P_{\alpha}^{f}(G, k) \\
& \quad= \begin{cases}k(k-1)(k-2)(k-3)(k-4), & \alpha=0 \\
k(k-1)(k-2)^{3}, & \alpha=0.2 \\
k^{2}(k-1)^{3}, & \alpha=0.3 \\
k^{5}, & \alpha=0.4 \\
k^{3}, & \alpha=0.6 \\
k, & \alpha=0.8\end{cases}
\end{aligned}
$$




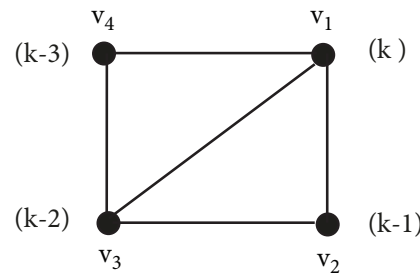

$(\mathrm{k}-2)$

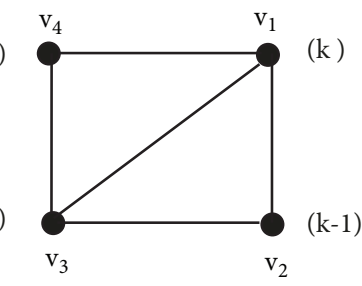

$\alpha=0 ; P_{\alpha}^{f}(G, k)=k(k-1)(k-2)(k-3)$

$(\mathrm{k}-1)$

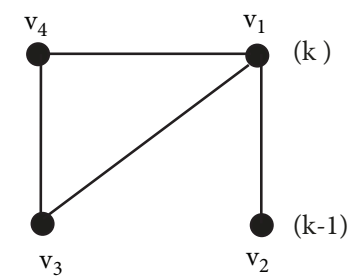

$\alpha=0.3 ; P_{\alpha}^{f}(G, k)=k(k-1)^{2}(k-2)$



$\alpha=0.8 ; P_{\alpha}^{f}(G, k)=k^{2}(k-1)^{2}$ $(\mathrm{k}-2)$

$\alpha=0.2 ; P_{\alpha}^{f}(G, k)=k(k-1)(k-2)^{2}$

(k-1)

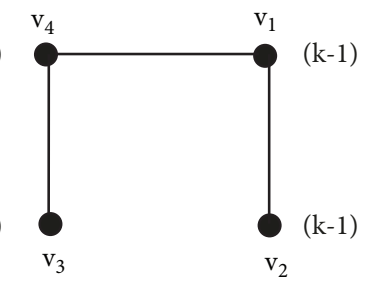

$\alpha=0.5 ; P_{\alpha}^{f}(G, k)=k(k-1)^{3}$

(k)

$\begin{array}{ll}\mathrm{v}_{1} & \\ \mathrm{C}^{\prime} & (\mathrm{k})\end{array}$

(k)



$\alpha=1 ; P_{\alpha}^{f}(G, k)=k^{4}$

FIGURE 2: Different fuzzy chromatic polynomials of the fuzzy graph $G$ in Example 23.

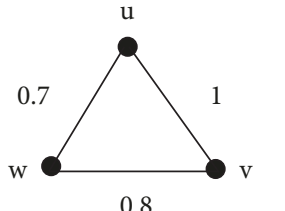

(a) A fuzzy graph $G$

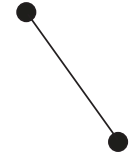

(b) A crisp graph $G_{\alpha}$ for $\alpha=1$

Figure 3

Remark 31. For fuzzy graph $G$ with fuzzy vertex set and fuzzy edge set, the degree of the fuzzy chromatic polynomial of $G$ is not always equal to the number of vertices of $G$. It can be easily seen from the fuzzy chromatic polynomial of the fuzzy graph in Example 29.

For instance, for $\alpha=0.4$, the degree of $P_{\alpha}^{f}(G, k)=5$ which is the number of vertices of $G$, but for $\alpha=0.6$, the degree of $P_{\alpha}^{f}(G, k)=3 \neq 5$; also for $\alpha=0.8$, the degree of $P_{\alpha}^{f}(G, k)=$ $1 \neq 5$.

Remark 32. The fuzzy chromatic polynomial of fuzzy graph $G$ with fuzzy vertex set and fuzzy edge set, $P_{\alpha}^{f}(G, k), k \geq \chi_{\alpha}$ will be decreased when the value of $\alpha$ will increase, since in fuzzy graph with fuzzy vertices and fuzzy edges, both $\left|\mathrm{V}_{\alpha}\right|$ and $\left|\mathrm{E}_{\alpha}\right|$ decrease as $\alpha$ increases (see Table 1).
Example 33. Consider a fuzzy graph $G$ with fuzzy vertex set and fuzzy edge set in Figure 6.

By routine computation, the fuzzy chromatic polynomial of the fuzzy graph $G$ in Figure 6 is obtained as

$$
P_{\alpha}^{f}(G, k)= \begin{cases}\mathrm{k}(\mathrm{k}-1)(\mathrm{k}-2), & \alpha=0 \\ \mathrm{k}(\mathrm{k}-1)(\mathrm{k}-2), & \alpha=0.1 \\ \mathrm{k}(\mathrm{k}-1)^{2}, & \alpha=0.5 \\ \mathrm{k}(\mathrm{k}-1), & \alpha=0.7 \\ k^{2}, & \alpha=0.8 \\ k, & \alpha=1\end{cases}
$$


TABLE 1: The values of $\alpha,\left|V_{\alpha}\right|,\left|E_{\alpha}\right|, \chi_{\alpha}$, and $P_{\alpha}^{f}(G, k), k \geq \chi_{\alpha}$ for the fuzzy graph $G$ in Example 33.

\begin{tabular}{|c|c|c|c|c|c|c|c|c|}
\hline$\alpha$ & $\left|\mathrm{V}_{\alpha}\right|$ & $\left|\mathrm{E}_{\alpha}\right|$ & $\chi_{\alpha}$ & $\mathrm{P}_{\alpha}^{\mathrm{f}}(\mathrm{G}, \mathrm{k})$ & $\mathrm{P}_{\alpha}^{\mathrm{f}}\left(\mathrm{G}, \chi_{\alpha}\right)$ & $\mathrm{P}_{\alpha}^{\mathrm{f}}\left(\mathrm{G}, \chi_{\alpha}+1\right)$ & $\mathrm{P}_{\alpha}^{\mathrm{f}}\left(\mathrm{G}, \chi_{\alpha}+2\right)$ & $\mathrm{P}_{\alpha}^{\mathrm{f}}\left(\mathrm{G}, \chi_{\alpha}+3\right)$ \\
\hline 0 & 3 & 3 & 3 & $\mathrm{k}(\mathrm{k}-1)(\mathrm{k}-2)$ & 6 & 24 & 60 & 120 \\
\hline 0.1 & 3 & 3 & 3 & $\mathrm{k}(\mathrm{k}-1)(\mathrm{k}-2)$ & 6 & 24 & 60 & 120 \\
\hline 0.5 & 3 & 2 & 2 & $\mathrm{k}(\mathrm{k}-1)^{2}$ & 2 & 12 & 36 & 80 \\
\hline 0.7 & 2 & 1 & 2 & $\mathrm{k}(\mathrm{k}-1)$ & 2 & 6 & 12 & 20 \\
\hline 0.8 & 2 & 0 & 1 & $\mathrm{k}^{2}$ & 1 & 4 & 9 & 16 \\
\hline 1 & 1 & 0 & 1 & $\mathrm{k}$ & 1 & 2 & 3 & 4 \\
\hline
\end{tabular}



Figure 4: A fuzzy graph $G$ with fuzzy vertices and fuzzy edges.

Table 1 shows that the fuzzy chromatic polynomial of the fuzzy graph $G$ in Example 33 deceases as $\alpha$ increases (see column 1 and columns 6-9 of Table 1). Moreover, both the number of vertices and the number of edges of $\alpha$-cut graph of the fuzzy graph decrease as $\alpha$ increases (see column 1 and columns 2 and 3 of Table 1 ).

\section{Main Results}

In this section, we prove some relevant results on fuzzy chromatic polynomial for fuzzy graphs which are discussed in Section 3. Moreover, the fuzzy chromatic polynomial of complete fuzzy graph and the fuzzy chromatic polynomial of fuzzy graphs which are cycles and fuzzy cycles are studied.

4.1. $\alpha$-Cut Graph and Fuzzy Graph. The relations among different $\alpha$-cuts of a fuzzy graph have been established as follows.

Lemma 34. Let $G$ be a fuzzy graph. If $0 \leq \alpha \leq \beta \leq 1$, then the following are true.

(i) $\left|V_{\alpha}\right| \geq\left|V_{\beta}\right|$

(ii) $\left|E_{\alpha}\right| \geq\left|E_{\beta}\right|$

Proof. Let $G=(V, \sigma, \mu)$ be a fuzzy graph and $0 \leq \alpha \leq \beta \leq 1$. Now, $G_{\alpha}=\left(V_{\alpha}, E_{\alpha}\right)$, where $V_{\alpha}=\{u \in V \mid \sigma(u) \geq \alpha\}$ and $E_{\alpha}=\{(u, v) \mid \mu(u, v) \geq \alpha\}$. Also, $G_{\beta}=\left(V_{\beta}, E_{\beta}\right)$, where $V_{\beta}=$ $\{u \in V \mid \sigma(u) \geq \beta\}$ and $E_{\beta}=\{(u, v) \mid \mu(u, v) \geq \beta\}$. Since $0 \leq \alpha \leq \beta \leq 1$, we have $V_{\beta} \subseteq V_{\alpha}$ and $E_{\beta} \subseteq E_{\alpha}$. From this, results (i) and (ii) hold.
Theorem 35. Let $G$ be a fuzzy graph. If $0 \leq \alpha \leq \beta \leq 1$, then $G_{\beta}$ is a subgraph of $G_{\alpha}$.

Proof. Let $G=(V, \sigma, \mu)$ be a fuzzy graph and $0 \leq \alpha \leq \beta \leq 1$. Now, $G_{\alpha}=\left(V_{\alpha}, E_{\alpha}\right)$, where $V_{\alpha}=\{u \in V \mid \sigma(u) \geq \alpha\}$ and $E_{\alpha}=\{(u, v) \mid \mu(u, v) \geq \alpha\}$. Also, $G_{\beta}=\left(V_{\beta}, E_{\beta}\right)$, where $V_{\beta}=$ $\{u \in V \mid \sigma(u) \geq \beta\}$ and $E_{\beta}=\{(u, v) \mid \mu(u, v) \geq \beta\}$. From Lemma 34, we get $V_{\beta} \subseteq V_{\alpha}$ and $E_{\beta} \subseteq E_{\alpha}$. That means, for any element $u \in V_{\beta}, u \in V_{\alpha}$ and for any element $(u, v) \in E_{\beta}$, $(u, v) \in E_{\alpha}$. Hence, $G_{\beta}$ is a subgraph of $G_{\alpha}$.

Theorem 36. Let $G$ be a fuzzy graph. If $0 \leq \alpha \leq \beta \leq 1$, then $\operatorname{deg} P\left(G_{\alpha}, k\right) \geq \operatorname{deg} P\left(G_{\beta}, k\right)$.

Proof. Let $G=(V, \sigma, \mu)$ be a fuzzy graph and $0 \leq \alpha \leq \beta \leq 1$. Now, $G_{\alpha}=\left(V_{\alpha}, E_{\alpha}\right)$, where $V_{\alpha}=\{u \in V \mid \sigma(u) \geq \alpha\}$ and $E_{\alpha}=\{(u, v) \mid \mu(u, v) \geq \alpha\}$. Also, $G_{\beta}=\left(V_{\beta}, E_{\beta}\right)$, where $V_{\beta}=$ $\{u \in V \mid \sigma(u) \geq \beta\}$ and $E_{\beta}=\{(u, v) \mid \mu(u, v) \geq \beta\}$. We know that the degree of the chromatic polynomial of a crisp graph is the number of vertices in the crisp graph. Therefore, the degree of $P\left(G_{\alpha}, k\right)=\left|V_{\alpha}\right|$ and the degree of $P\left(G_{\beta}, k\right)=$ $\left|V_{\beta}\right|$. By Lemma 34(i), we have $\left|V_{\alpha}\right| \geq\left|V_{\beta}\right|$. Therefore, deg $P\left(G_{\alpha}, k\right)=\left|V_{\alpha}\right| \geq\left|V_{\beta}\right|=\operatorname{deg} P\left(G_{\beta}, k\right)$.

The relations between the $\alpha$-cut graph of a fuzzy graph and the value of $\alpha=0$, the fuzzy chromatic polynomial of a fuzzy graph, and the chromatic polynomial of corresponding complete crisp graph can be determined below.

Lemma 37. Let $G$ be a fuzzy graph with $n$ vertices and $G_{\alpha}$ be $\alpha$-cut of $G$. Then if $\alpha=0$, then $G_{\alpha}$ is a complete crisp graph with $n$ vertices.

Proof. Let $G=(V, \sigma, \mu)$ be a fuzzy graph with n vertices and $\alpha=0$. Now, $G_{0}=\left(V_{0}, E_{0}\right)$, where $V_{0}=\{u \in V \mid \sigma(u) \geq 0\}$ and $E_{0}=\{(u, v) \mid \mu(u, v) \geq 0\}$. Here, $V_{0}$ consists of all the vertices in V of G. Similarly, $E_{0}$ consists of all the edges in E and all the edges not in $\mathrm{E}$ of $\mathrm{G}$. This shows that all the vertices in $V_{0}$ of $G_{0}$ are adjacent to each other. Therefore, $G_{0}$ is a complete crisp graph of $\mathrm{n}$ vertices. This completes the proof.

Remark 38. For $\alpha$-cut of the fuzzy graph $G$ with $n$ vertices, $G_{\alpha}=K_{n}$ if $\alpha=0$.

Theorem 39. Let $G$ be a fuzzy graph with $n$ vertices and $G_{\alpha}$ be $\alpha$-cut of $G$. Then if $\alpha=0$,

$$
P_{\alpha}^{f}(G, k)=P\left(K_{n}, k\right)
$$




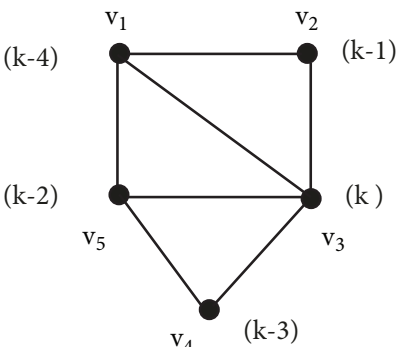

$\alpha=0 ; P_{\alpha}^{f}(G, k)=k(k-1)(k-2)(k-3)(k-4)$

(k-1)

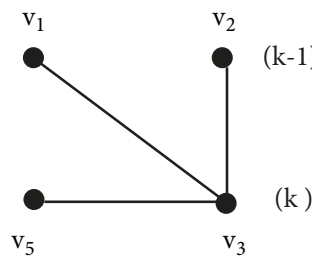

(k)

$\alpha=0.3 ; P_{\alpha}^{f}(G, k)=k^{2}(k-1)^{3}$

$\mathrm{v}_{2}$

- $(\mathrm{k})$

$\mathrm{v}_{5}$

( k)

\section{(k)}

(k)

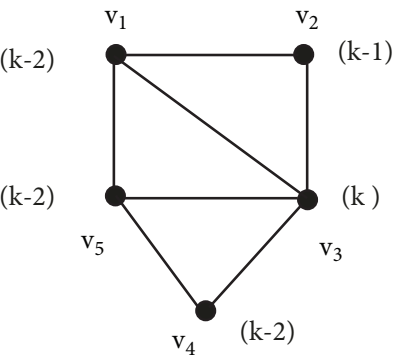

$\alpha=0.2 ; P_{\alpha}^{f}(G, k)=k(k-1)(k-2)^{3}$

(k)

$\mathrm{v}_{2}(\mathrm{k})$

(k)

- $\mathrm{v}_{3}$

(k)

$$
\alpha=0.4 ; P_{\alpha}^{f}(G, k)=k^{5}
$$

$(\mathrm{k})$

$\mathrm{v}_{5}$

( k )

$\alpha=0.8 ; P_{\alpha}^{f}(G, k)=k$

Figure 5: Different fuzzy chromatic polynomials of the fuzzy graph G in Example 29.

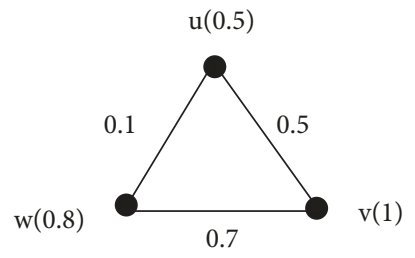

Figure 6: A fuzzy graph G.

Proof. Let $G=(V, \sigma, \mu)$ be a fuzzy graph with $\mathrm{n}$ vertices and $\alpha=0$. Now, $G_{\alpha}=\left(V_{\alpha}, E_{\alpha}\right)$, where $V_{\alpha}=\{u \in V \mid \sigma(u) \geq \alpha\}$ and $E_{\alpha}=\{(u, v) \mid \mu(u, v) \geq \alpha\}$. Since $G$ is fuzzy graph. Then by Definition 20 and Remark 38, the result holds.

The relation between fuzzy chromatic polynomial of a fuzzy graph and the chromatic polynomial of corresponding underlying crisp graph is established below.

Theorem 40. Let $G$ be a fuzzy graph and $G^{*}$ be its underlying crisp graph. If $\alpha=\min (L)$, where $L$ is the level set or fundamental set of $G$, then $P_{\alpha}^{f}(G, k)=P\left(G^{*}, k\right)$.

Proof. Let $G=(V, \sigma, \mu)$ be a fuzzy graph and let $G^{*}=$ $\left(V^{*}, E^{*}\right)$ be the underlying crisp graph of $G$, where $V^{*}=\{u \in$
$V \mid \sigma(u)>0\}$ and $E^{*}=\{(u, v), u, v \in V \mid \mu(u, v)>0\}$. This means that $V^{*}=V$ and $E^{*}$ consists of all the edges in E. Let $L=\left\{\alpha_{1}, \alpha_{2}, \ldots, \alpha_{k}\right\}$ be a level set or fundamental set of G. Put $\alpha=\min (L)$; now $G_{\alpha}=\left(V_{\alpha}, E_{\alpha}\right)$, where $V_{\alpha}=\{u \in$ $V \mid \sigma(u) \geq \alpha\}$ and $E_{\alpha}=\{(u, v) \mid \mu(u, v) \geq \alpha\}$. Since $\alpha$ is the minimum value in the level set $\mathrm{L}, V_{\alpha}$ consists of all the vertices of $\mathrm{V}$ and $E_{\alpha}$ consists of all the edges in E. Therefore, $G_{\alpha}=G^{*}$. Since $G$ is fuzzy graph, then, by Definition 20, we have $P_{\alpha}^{f}(G, k)=P\left(G_{\alpha}, k\right)=P\left(G^{*}, k\right)$.

The following is an important theorem for computing the fuzzy chromatic polynomial of a fuzzy graph explicitly.

Theorem 41. Let $G$ be a fuzzy graph with $n$ vertices and $G^{*}$ be its underlying crisp graph. If $\alpha \in I=L \cup\{0\}$ and $\beta=\min (L)$, then

$$
P_{\alpha}^{f}(G, k)= \begin{cases}P\left(K_{n}, k\right) & \text { if } \alpha=0 \\ P\left(G^{*}, k\right) & \text { if } \alpha=\beta \\ P\left(G_{\alpha}, k\right) & \text { if } \beta<\alpha \leq 1\end{cases}
$$

where $K_{n}$ is a complete crisp graph with $n$ vertices.

Proof. Let $G=(V, \sigma, \mu)$ be a fuzzy graph with $\mathrm{n}$ vertices and let $G^{*}=\left(V^{*}, E^{*}\right)$ be the underlying crisp graph of $G$, where 
$V^{*}=\{u \in V \mid \sigma(u)>0\}$ and $E^{*}=\{(u, v), u, v \in V \mid \mu(u, v)>$ $0\}$. For $\alpha \in \mathrm{I}$, now $G_{\alpha}=\left(V_{\alpha}, E_{\alpha}\right)$, where $V_{\alpha}=\{u \in V \mid \sigma(u) \geq$ $\alpha\}$ and $E_{\alpha}=\{(u, v) \mid \mu(u, v) \geq \alpha\}$. Let us prove the theorem by considering three cases.

Case 1 . Let $\alpha=0$. Then by Theorem 39, the fuzzy chromatic polynomial of the fuzzy graph $G$ is the chromatic polynomial of a complete crisp graph with $n$ vertices, $K_{n}$. That is,

$$
P_{\alpha}^{f}(G, k)=P\left(K_{n}, k\right)
$$

Case 2. Let $\alpha=\beta$, where $\beta=\min (L)$. Then by Theorem 40, the fuzzy chromatic polynomial of the fuzzy graph $G$ is the chromatic polynomial of its underlying crisp graph $G^{*}$. That is,

$$
P_{\alpha}^{f}(G, k)=P\left(G^{*}, k\right)
$$

Case 3. Let $\beta<\alpha \leq 1$; since $\alpha$ is in I, then by Definition 20 $P_{\alpha}^{f}(G, k)=P\left(G_{\alpha}, k\right)$. This completes the proof.

The following result shows that the degree of fuzzy chromatic polynomial of a fuzzy graph is less than or equal to the number of vertices of the fuzzy graph. It is a significant difference from crisp graph theory.

Theorem 42. Let $G=(V, \sigma, \mu)$ be a fuzzy graph. Then the degree of $P_{\alpha}^{f}(G, k) \leq|V|, \forall \alpha \in I$.

Proof. Let us prove the theorem by considering two cases.

Case 1. Let $G=(V, \mu)$ be a fuzzy graph with crisp vertices and fuzzy edges. By Lemma 28, the degree of $P_{\alpha}^{f}(G, k)=|V|, \forall \alpha \in$ I. Therefore, the result holds.

Case 2. Let $G=(V, \sigma, \mu)$ be a fuzzy graph with fuzzy vertex set and fuzzy edge set. Now, $G_{\alpha}=\left(V_{\alpha}, E_{\alpha}\right)$, where $V_{\alpha}=$ $\{u \in V \mid \sigma(u) \geq \alpha\}$ and $E_{\alpha}=\{(u, v) \mid \mu(u, v) \geq \alpha\}$. We know that the degree of the chromatic polynomial of a crisp graph $G_{\alpha}$ is the number of vertices of $G_{\alpha}$. That is, the degree of $P\left(G_{\alpha}, k\right)=\left|V_{\alpha}\right|$. But for all $\alpha \in I,\left|V_{\alpha}\right| \leq|V|$, since $V_{\alpha} \subseteq \mathrm{V}$. Then by Definition 20, we have $P_{\alpha}^{f}(G, k)=P\left(G_{\alpha}, k\right)$, for $\alpha \in I$. Therefore, the degree of $P_{\alpha}^{f}(G, k)=$ the degree of $P\left(G_{\alpha}, k\right)=$ $\left|V_{\alpha}\right| \leq|V|$. This implies that $P_{\alpha}^{f}(G, k) \leq|V|, \forall \alpha \in I$. Hence, from Case 1 and Case 2, we conclude that, for any fuzzy graph $G$, the degree of $P_{\alpha}^{f}(G, k) \leq|V|, \forall \alpha \in I$.

4.2. Complete Fuzzy Graph and Fuzzy Cycle. In 1989, Bhutani [11] introduced the concept of a complete fuzzy graph as follows.

Definition 43 (see [11]). A complete fuzzy graph is a fuzzy graph $G=(V, \sigma, \mu)$ such that $\mu(u, v)=\sigma(u) \wedge \sigma(v)$ for all $u, v \in V$.

The following result gives the underlying crisp graph of a complete fuzzy graph is a complete crisp graph.
Lemma 44. Let $G$ be a complete fuzzy graph and $G^{*}$ be its underlying crisp graph. Then $G^{*}$ is a complete crisp graph.

It is clear from the following example.

Example 45. We show that the underlying crisp graph of a complete fuzzy graph is complete crisp graph. Let $V=$ $\{u, v, w, x\}$. Define the fuzzy set $\sigma$ on $\mathrm{V}$ as $\sigma(u)=0.7, \sigma(v)=$ $0.8, \sigma(w)=1$, and $\sigma(x)=0.6$. Define a fuzzy set $\mu$ on E such that $\mu(u, v)=\mu(u, w)=0.7, \mu(u, x)=\mu(v, x)=$ $\mu(w, x)=0.6$, and $\mu(v, w)=0.8$. Then $\mu(u, v)=\sigma(u) \wedge \sigma(v)$ for all $u, v \in V$. Thus, $G=(V, \sigma, \mu)$ is a complete fuzzy graph. Now, $G^{*}=\left(V^{*}, E^{*}\right)$, where $V^{*}=\{u \in V \sigma(u)>$ $0\}=\{u, v, w, x\}$ and $E^{*}=\{(u, v), u, v \in V \mu(u, v)>$ $0\}=\{(u, v),(u, w),(u, x),(v, x),(w, x),(v, w)\}$. This shows that each vertex in $G^{*}$ joins each of the other vertices in $G^{*}$ exactly by one edge. Therefore, $G^{*}$ is a complete crisp graph $K_{4}$.

Remark 46. For complete fuzzy graph $G$ with $n$ vertices, by Lemma $44, G^{*}=K_{n}$, where $K_{n}$ is a complete crisp graph.

The following is an important theorem for computing the fuzzy chromatic polynomial of a complete fuzzy graph explicitly.

Theorem 47. Let $G$ be a complete fuzzy graph with $n$ vertices. If $\alpha \in I=L \cup\{0\}$ and $\beta=\min (L)$ then

$$
P_{\alpha}^{f}(G, k)= \begin{cases}P\left(K_{n}, k\right) & \text { if } \alpha=0 \text { and } \beta \\ P\left(G_{\alpha}, k\right) & \text { if } \beta<\alpha \leq 1\end{cases}
$$

Proof. Let $G=(V, \sigma, \mu)$ be a complete fuzzy graph with $\mathrm{n}$ vertices and let $G^{*}=\left(V^{*}, E^{*}\right)$ be the underlying crisp graph of $G$, where $V^{*}=\{u \in V \mid \sigma(u)>0\}$ and $E^{*}=\{(u, v), u, v \in$ $V \mid \mu(u, v)>0\}$. For $\alpha \in \mathrm{I}=\mathrm{L} \cup\{0\}$, where $\mathrm{L}$ is a level set of $G$ now $G_{\alpha}=\left(V_{\alpha}, E_{\alpha}\right)$ where $V_{\alpha}=\{u \in V \mid \sigma(u) \geq \alpha\}$ and $E_{\alpha}=\{(u, v) \mid \mu(u, v) \geq \alpha\}$. Let us prove the theorem by considering three cases.

Case 1. Let $\alpha=0$, and since $G$ is a fuzzy graph with $\mathrm{n}$ vertices then, by Theorem 39, we have $P_{\alpha}^{f}(G, k)=P\left(K_{n}, k\right)$.

Case 2. Let $\alpha=\beta$, where $\beta=\min (L)$; since $G$ is a fuzzy graph then, by Theorem 40 , we have

$$
P_{\alpha}^{f}(G, k)=P\left(G^{*}, k\right)
$$

and since $G$ is complete fuzzy graph, then, by Remark 46 and (11), we get $P_{\alpha}^{f}(G, k)=P\left(K_{n}, k\right)$.

Case 3. Let $\beta<\alpha \leq 1$; since $\alpha$ is in I and $G$ is a fuzzy graph then by Theorem $41 P_{\alpha}^{f}(G, k)=P\left(G_{\alpha}, k\right)$. This completes the proof.

Remark 48. For a complete fuzzy graph $G=(V, \sigma, \mu)$ with crisp vertices (i.e., $\sigma(v)=1, \forall v \in V)$ and $|V|=n, P_{\alpha}^{f}(G, k)=$ $P\left(K_{n}, k\right)$ for all $\alpha \in \mathrm{I}$. 
The fuzzy chromatic polynomial of a complete fuzzy graph with $|L|=|V|$ can be defined in terms of the chromatic polynomial of complete crisp graph as follows.

Definition 49. Let $G=(V, \sigma, \mu)$ be a complete fuzzy graph with $|V|=n$ vertices. Let $L$ be a level set of $G$ and $\alpha \in I=$ $L U\{0\}$. Define $\beta_{0}=\min (L)=\beta, \beta_{1}=\min \left(L \backslash\left\{\beta_{0}\right\}\right), \beta_{2}=$ $\min \left(L \backslash\left\{\beta_{0}, \beta_{1}\right\}\right), \ldots, \beta_{n-1}=\min \left(L \backslash\left\{\beta_{0}, \beta_{1}, \ldots, \beta_{n-2}\right\}\right)$.

If $|L|=|V|=n, n \geq 1$, then the fuzzy chromatic polynomial of a complete fuzzy graph $G$ is defined as

$$
P_{\alpha}^{f}(G, k)= \begin{cases}P\left(K_{n}, k\right) & \text { if } \alpha=0 \& \beta_{0} \\ P\left(K_{n-1}, k\right) & \text { if } \alpha=\beta_{1} \\ P\left(K_{n-2}, k\right) & \text { if } \alpha=\beta_{2} \\ \vdots & \vdots \\ P\left(K_{1}, k\right) & \text { if } \alpha=\beta_{n-1}\end{cases}
$$

where $K_{n}, n \geq 1$ is a complete crisp graph with $\mathrm{n}$ vertices.

Note that the advantage of Definition 49 is that the fuzzy chromatic polynomial of a complete fuzzy graph with $|V|=$ $|L|$ can be obtained without using the formal procedures. This situation can be illustrated in the following example.

Example 50. Consider the complete fuzzy graph $G$ in Figure 7.

In Figure 7, $|V|=4, I=L \cup\{0\}$, where $\mathrm{L}=\{0.6,0.7,0.8,1\}$. Therefore, $|L|=4$. Also, $\beta_{0}=0.6, \beta_{1}=0.7, \beta_{2}=0.8$, and $\beta_{3}=1$.

Since $|L|=|V|=4$, then, by Definition 49 , the fuzzy chromatic polynomial of the complete fuzzy graph $G$ is

$$
P_{\alpha}^{f}(G, k)= \begin{cases}k(k-1)(k-2)(k-3), & \alpha=0 \& 0.6 \\ k(k-1)(k-2), & \alpha=0.7 \\ k(k-1), & \alpha=0.8 \\ k, & \alpha=1\end{cases}
$$

A fuzzy graph is a cycle and a fuzzy cycle as defined in $[9,32]$.

Definition 51 (see $[9,32])$. Let $G=(V, \sigma, \mu)$ be a fuzzy graph. Then

(i) $G$ is called a cycle if $G^{*}=\left(V^{*}, E^{*}\right)$ is a cycle;

(ii) $G$ is called a fuzzy cycle if $G^{*}=\left(V^{*}, E^{*}\right)$ is a cycle and $\nexists$ unique $(x, y) \in E^{*}$ such that $\mu(x, y)=\wedge\{\mu(u, v) \mid$ $\left.(u, v) \in E^{*}\right\}$.

Remark 52. If a fuzzy graph $G$ is cycle with $\mathrm{n}$ vertices, then $G^{*}=C_{n}$, where $C_{n}$ is a cycle crisp graph with n vertices.

The following theorem is important for computing the fuzzy chromatic polynomial of a fuzzy graph which is cycle.

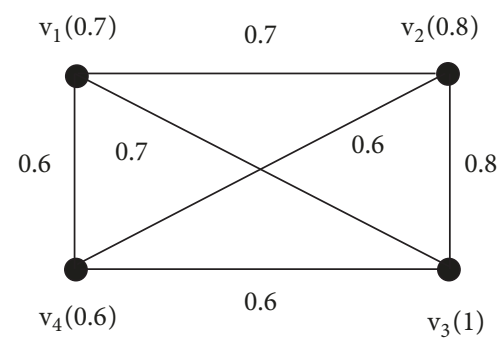

FIgURE 7: A complete fuzzy graph $G$ with $|V|=|L|$.

Theorem 53. Let $G$ be a fuzzy graph with $n$ vertices. Then $G$ is a cycle if and only if

$$
P_{\alpha}^{f}(G, k)= \begin{cases}P\left(K_{n}, k\right) & \text { if } \alpha=0 \\ P\left(C_{n}, k\right) & \text { if } \alpha=\beta \\ P\left(G_{\alpha}, k\right) & \text { if } \beta<\alpha \leq 1\end{cases}
$$

where $K_{n}$ and $C_{n}$ are a complete crisp graph and cycle crisp graph with $n$ vertices, respectively.

Proof. Let $G=(V, \sigma, \mu)$ be a fuzzy graph with $\mathrm{n}$ vertices. Suppose $G$ be a cycle. Since $G$ is a cycle, $G^{*}$ is a cycle crisp graph. For $\alpha \in \mathrm{I}=\mathrm{L} \cup\{0\}$, where $\mathrm{L}$ is a level set of $G$, now $G_{\alpha}=\left(V_{\alpha}, E_{\alpha}\right)$, where $V_{\alpha}=\{u \in V \mid \sigma(u) \geq \alpha\}$ and $E_{\alpha}=\{(u, v) \mid \mu(u, v) \geq \alpha\}$. Let us prove the theorem by considering three cases.

Case 1. Let $\alpha=0$, and since $G$ is a fuzzy graph with $\mathrm{n}$ vertices then by Theorem 39, we have $P_{\alpha}^{f}(G, k)=P\left(K_{n}, k\right)$.

Case 2. Let $\alpha=\beta$, where $\beta=\min (L)$; since $G$ is a fuzzy graph then, by Theorem 40 , we have

$$
P_{\alpha}^{f}(G, k)=P\left(G^{*}, k\right)
$$

and since $\mathrm{G}$ is cycle with $\mathrm{n}$ vertices, then, by Remark 52 and (15), we get $P_{\alpha}^{f}(G, k)=P\left(C_{n}, k\right)$.

Case 3. Let $\beta<\alpha \leq 1$; since $\alpha$ is in I and $G$ is a fuzzy graph then, by Theorem $41, P_{\alpha}^{f}(G, k)=P\left(G_{\alpha}, k\right)$. Hence, the result holds.

Conversely, we shall prove it by contrapositive. Suppose $G$ is not a cycle. Then, by Definition $51, G^{*}$ is not a cycle. This implies that $G^{*} \neq C_{n}$. Hence,

$$
P_{\alpha}^{f}(G, k) \neq \begin{cases}P\left(K_{n}, k\right) & \text { if } \alpha=0 \\ P\left(C_{n}, k\right) & \text { if } \alpha=\beta \\ P\left(G_{\alpha}, k\right) & \text { if } \beta<\alpha \leq 1\end{cases}
$$

Therefore, if (14) is true, then $G$ is a cycle. This completes the proof.

Corollary 54. Let $G$ be a fuzzy graph. If $G$ is a fuzzy cycle with $n$ vertices; then (14) is true. 


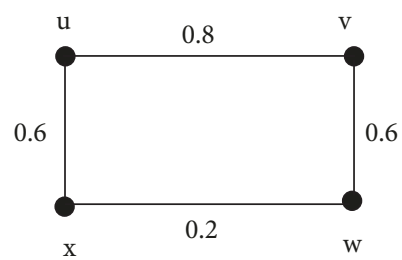

Figure 8: A fuzzy graph G.

Proof. Let $G$ be a fuzzy cycle with $n$ vertices. Since $G$ is fuzzy cycle, then its $G^{*}$ is cycle. Then by Definition $51, G$ is a cycle. Then by Theorem 53, the result is immediate.

Remark 55. The converse of Corollary 54 is not true. It can be seen from the following example.

Example 56. Consider the fuzzy graph $G$ given in Figure 8.

In Figure 8 , clearly $G^{*}$ is a cycle. So, $G$ is cycle. For $\alpha \epsilon$ $I=L \cup\{0\}$, where $L=\{0.2,0.6,0.8,1\}$, since $G$ is cycle, then, by (14), the fuzzy chromatic polynomial of $G$ is

$$
P_{\alpha}^{f}(G, k)= \begin{cases}P\left(K_{4}, k\right) & \text { if } \alpha=0 \\ P\left(C_{4}, k\right) & \text { if } \alpha=0.2 \\ P\left(G_{\alpha}, k\right) & \text { if } 0.2<\alpha \leq 1\end{cases}
$$

But $G$ is not fuzzy cycle.

Remark 57. In general, the meaning of fuzzy chromatic polynomial of a fuzzy graph depends on the sense of the index $\alpha$. It can be interpreted that for lower values of $\alpha$ more numbers of different k-colorings are obtained. On the other hand, for higher values of $\alpha$ less number of different $\mathrm{k}$-colorings are obtained. Therefore, the fuzzy chromatic polynomial sums up all this information so as to manage the fuzzy problem.

\section{Conclusions}

In this paper, the concept of fuzzy chromatic polynomial of fuzzy graph with crisp and fuzzy vertex sets is introduced. The fuzzy chromatic polynomial of fuzzy graph is defined based on $\alpha$-cuts of the fuzzy graph. Some properties of the fuzzy chromatic polynomial of fuzzy graphs are investigated. The degree of fuzzy chromatic polynomial of a fuzzy graph is less than or equal to the number of vertices in fuzzy graph which is a significant difference from the crisp graph theory. Further, the fuzzy chromatic polynomial of fuzzy graph with fuzzy vertex set will decrease when the value of $\alpha$ will increase. Also, more results on fuzzy chromatic polynomial of fuzzy graphs have been proved. The relation between the fuzzy chromatic polynomial of fuzzy graph and its underlying crisp graph and the relation between the fuzzy chromatic polynomial of fuzzy graph and complete crisp graph are established. Finally, the fuzzy chromatic polynomial for complete fuzzy graph and fuzzy cycle is studied. Also, the fuzzy chromatic polynomial of complete fuzzy graph is defined explicitly when $|V|=|L|$.
We are working on fuzzy chromatic polynomial based on different types of fuzzy coloring functions as an extension of this study.

\section{Data Availability}

The data used to support the findings of this study are included within the article.

\section{Conflicts of Interest}

The authors declare that there are no conflicts of interest regarding the publication of this paper.

\section{Authors' Contributions}

Both authors contributed equally and significantly in writing this article. Both authors read and approved the final manuscript.

\section{References}

[1] L. A. Zadeh, "Fuzzy sets," Information and Computation, vol. 8, pp. 338-353, 1965.

[2] C. M. Klein, "Fuzzy shortest paths," Fuzzy Sets and Systems, vol. 39, no. 1, pp. 27-41, 1991.

[3] K. C. Lin and M. S. Chern, "The fuzzy shortest path problem and its most vital arcs," Fuzzy Sets and Systems, vol. 58, no. 3, pp. 343-353, 1993.

[4] S. Okada and T. Soper, "A shortest path problem on a network with fuzzy arc lengths," Fuzzy Sets and Systems, vol. 109, no. 1, pp. 129-140, 2000.

[5] S. M. Nayeem and M. Pal, "Shortest path problem on a network with imprecise edge weight," Fuzzy Optimization and Decision Making, vol. 4, no. 4, pp. 293-312, 2005.

[6] A. Dey, A. Pal, and T. Pal, "Interval type 2 fuzzy set in fuzzy shortest path problem," Mathematics, vol. 4, no. 4, pp. 1-19, 2016.

[7] A. Dey, R. Pradhan, A. Pal, and T. Pal, "A genetic algorithm for solving fuzzy shortest path problems with interval type-2 fuzzy arc lengths," Malaysian Journal of Computer Science, vol. 31, no. 4, pp. 255-270, 2018.

[8] A. Kaufmann, Introduction A La Theorie Des Sous-Ensembles Flous, vol. 1, Masson et Cie, Paris, 1973.

[9] A. Rosenfeld, "Fuzzy graphs," in Fuzzy Sets and Their Applications, L. A. Zadeh, K. S. Fu, and M. Shimura, Eds., pp. 77-95, Academic Press, New York, NY, USA, 1975.

[10] P. Bhattacharya, "Some remarks on fuzzy graphs," Pattern Recognition Letters, vol. 6, no. 5, pp. 297-302, 1987.

[11] K. R. Bhutani, “On automorphisms of fuzzy graphs," Pattern Recognition Letters, vol. 9, no. 3, pp. 159-162, 1989.

[12] J. N. Mordeson and P. S. Nair, "Cycles and cocycles of fuzzy graphs," Information Sciences, vol. 90, no. 1-4, pp. 39-49, 1996.

[13] M. S. Sunitha and A. Vijayakumar, "A characterization of fuzzy trees," Information Sciences, vol. 113, no. 3-4, pp. 293-300, 1999.

[14] M. S. Sunitha and A. Vijaya Kumar, "Complement of a fuzzy graph," Indian Journal of Pure and Applied Mathematics, vol. 33, no. 9, pp. 1451-1464, 2002.

[15] K. R. Bhutani and A. Rosenfeld, "Strong arcs in fuzzy graphs," Information Sciences, vol. 152, pp. 319-322, 2003. 
[16] S. Mathew and M. S. Sunitha, "Types of arcs in a fuzzy graph," Information Sciences, vol. 179, no. 11, pp. 1760-1768, 2009.

[17] M. Akram, "Bipolar fuzzy graphs," Information Sciences, vol. 181, no. 24, pp. 5548-5564, 2011.

[18] M. Akram and W. A. Dudek, "Interval-valued fuzzy graphs," Computers \& Mathematics with Applications, vol. 61, no. 2, pp. 289-299, 2011.

[19] J. N. Mordeson and P. S. Nair, Fuzzy Graphs and Fuzzy Hypergraphs, Physica-Verlag, Heidelberg, Germany, 2000.

[20] M. Ananthanarayanan and S. Lavanya, "Fuzzy graph coloring using $\alpha$ cuts," International Journal of Engineering and Applied Sciences, vol. 4, no. 10, pp. 23-28, 2014.

[21] C. Eslahchi and B. N. Onagh, "Vertex-strength of fuzzy graphs," International Journal of Mathematics and Mathematical Sciences, vol. 2006, Article ID 43614, 9 pages, 2006.

[22] S. Muñoz, M. T. Ortuño, J. Ramírez, and J. Yáñez, "Coloring fuzzy graphs," Omega , vol. 33, no. 3, pp. 211-221, 2005.

[23] A. Kishore and M. S. Sunitha, "Chromatic number of fuzzy graphs," Annals of Fuzzy Mathematics and Informatics, vol. 7, no. 4, pp. 543-551, 2014.

[24] A. Dey and A. Pal, "Vertex coloring of a fuzzy graph using alph cut," International Journal of Management, vol. 2, no. 8, pp. 340352, 2012.

[25] A. Dey and A. Pal, "Fuzzy graph coloring technique to classify the accidental zone of a traffic control," Annals of Pure and Applied Mathematics, vol. 3, no. 2, pp. 169-178, 2013.

[26] A. Dey, R. Pradhan, A. Pal, and T. Pal, "The fuzzy robust graph coloring problem," in Proceedings of the 3rd International Conference on Frontiers of Intelligent Computing:Theory and Applications (FICTA) 2014, vol. 327, pp. 805-813, Springer, 2015.

[27] I. Rosyida, Widodo, C. R. Indrati, and K. A. Sugeng, "A new approach for determining fuzzy chromatic number of fuzzy graph," Journal of Intelligent and Fuzzy Systems. Applications in Engineering and Technology, vol. 28, no. 5, pp. 2331-2341, 2015.

[28] S. Samanta, T. Pramanik, and M. Pal, "Fuzzy colouring of fuzzy graphs," Afrika Matematika, vol. 27, no. 1-2, pp. 37-50, 2016.

[29] A. Dey, L. H. Son, P. K. K. Kumar, G. Selvachandran, and S. G. Quek, "New concepts on vertex and edge coloring of simple vague graphs," Symmetry, vol. 10, no. 9, pp. 1-18, 2018.

[30] R. A. Brualdi, Introductory Combinatorics, Prentice Hall, 2009.

[31] R. J. Wilson, Introduction to Graph Theory, Pearson, England, 2010.

[32] S. Mathew, J. N. Mordeson, and D. S. Malik, "Fuzzy graphs," in Fuzzy Graph Theory, vol. 363, pp. 13-83, Springer International Publishing AG, 2018. 




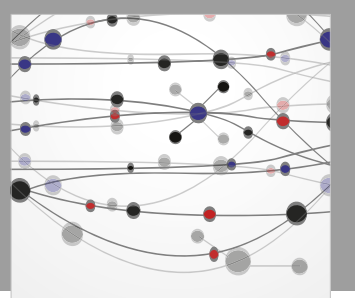

The Scientific World Journal
Submit your manuscripts at

Computing


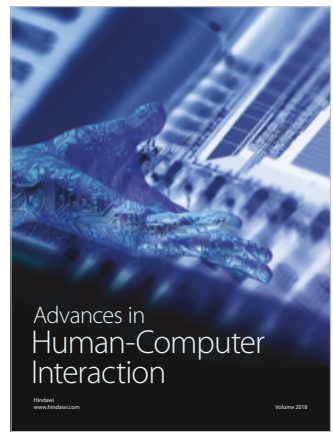

Human-Compute

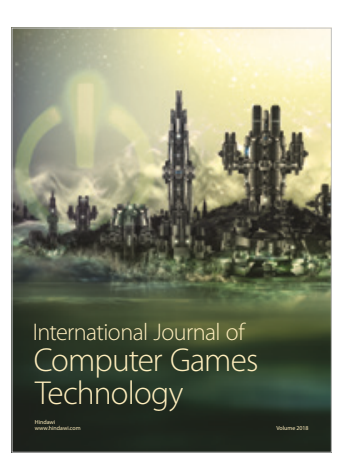

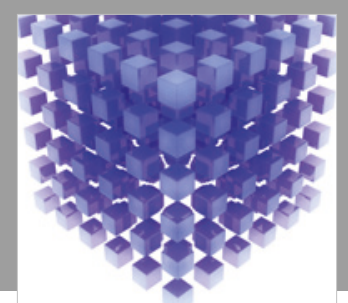

Mathematical Problems in Engineering

\section{Engincering}


www.hindawi.com


Computer Networks and Communications

Multimedia
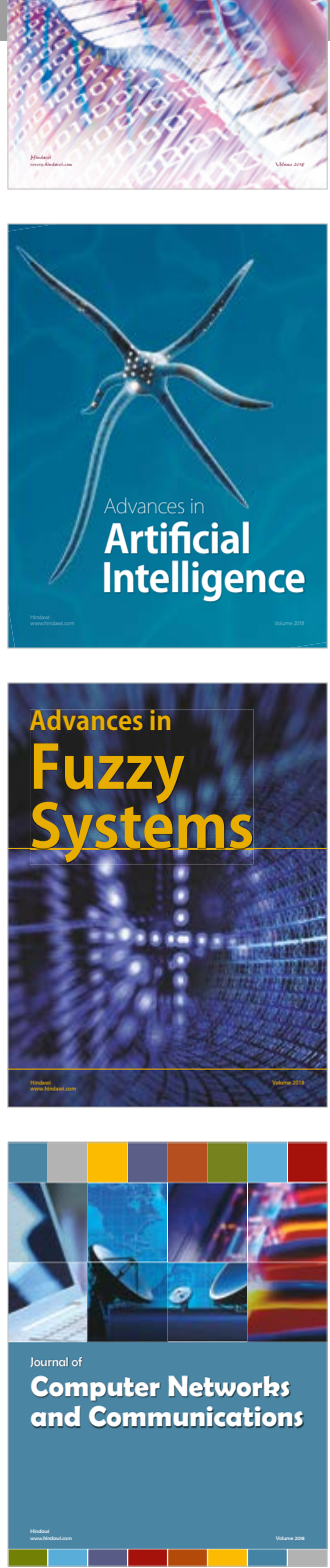

Advances in

Modelling \&

Simulation

in Engineering

interaction





